\title{
Aggressive Angiomyxoma with Diffusion-Weighted Magnetic Resonance Imaging and Dynamic Contrast Enhancement: A Case Report and Review of the Literature
}
S. Brunelle
F. Bertucci
B. Chetaille
B. Lelong
G. Piana
A. Sarran

Institut Paoli-Calmettes, Marseille, France

\section{Key Words}

Aggressive angiomyxoma · Magnetic resonance imaging · Diffusion-weighted imaging · Dynamic contast enhancement

\begin{abstract}
Introduction: Aggressive angiomyxoma (AA) is a rare benign soft tissue tumour usually affecting the pelvis and perineum of young women. Magnetic resonance imaging (MRI) is crucial in the management of AA patients for its diagnostic contribution and for the preoperative assessment of the actual tumour extension. Given the current development of less aggressive therapeutics associated with a higher risk of recurrence, close follow-up with MRI is fundamental after treatment. In this context, diffusion-weighted (DW) imaging has already shown high efficacy in the detection of early small relapses in prostate or rectal cancer. Case Report: We report here a case of pelvic AA in a 51-year-old woman examined with dynamic contrast enhancement and DW-MRI, including apparent diffusion coefficient mapping and calculation. Conclusion: To our knowledge, this is the first description of DWMRI in AA reported in the literature. Here, knowledge about imaging features of AA will be reviewed and expanded.




\section{Introduction}

Aggressive angiomyxoma (AA) is a benign soft tissue tumour with myxoid and vascular components that arises mostly in the lower pelvis and perineum in women of reproductive age. It is a very rare tumour. Since its first description in 1983, about 250 cases have been reported in the English literature. Most of them ( $>90 \%$ ) occurred in young women, usually in the second to fourth decade [1]. AA is defined as benign but has infiltrative potential into skeletal muscle and fat. The risks of local extension and postoperative local relapse justify surgical excision of the tumour.

Imaging and particularly magnetic resonance imaging (MRI) are fundamental for the clinical management of AA [2]. MRI findings are very characteristic and suggestive of the diagnosis. Precise preoperative assessment of local extension is crucial to plan the best surgical approach for tumour removal involving as little mutilation as possible. MRI allows the monitoring of the response to possible preoperative treatment such as hormone therapy. Finally, it is also crucial during the patient's follow-up after initial treatment.

For a few years now, classical MRI coupled with diffusion-weighted (DW) sequences has developed in oncology, leading to improvements in the detection and characterization of tumours, treatment response monitoring, and detection of recurrence [3, 4]. To our knowledge, we report here the first case of pelvic AA explored with DW-MRI and dynamic contrast enhancement (DCE)-MRI.

\section{Case Report}

In July 2011, a 51-year-old woman presented to her general practitioner with an incidentally discovered renitent mass in the internal part of the left buttock. Initial ultrasound (US) showed a well-circumscribed mass initially evoking a lipoma. Due to the large volume of the mass, the patient was referred to our hospital.

The patient did not have any specific medical, personal, or familial history. Her treatment included hormone replacement therapy. Rectal examination palpated the renitent mass. She was otherwise asymptomatic with a performance status score of 0 on the WHO scale. Laboratory findings were unremarkable. US showed a superficial, soft, hypoechoic, well-limited, solid mass. US-guided biopsy showed mature adipocytes associated with nonatypical fibroblast-like cells. Endorectal US showed a 39-mm hypoechoic lesion distant from the internal and external sphincters.

MRI showed a bulky perineal and intrapelvic tumour displacing rather than infiltrating the vagina, anal canal, and rectal structures. The mass was well limited, hourglass-shaped and had a maximum diameter of $11 \mathrm{~cm}$ (fig. 1). On T1-weighted imaging, the tumour was isointense relative to muscle. T2-weighted imaging showed typical high signal intensity with layered strands of lower signal intensity and swirled architecture. Finger-like projections into the underlying adipose tissue were observed. A fat linear structure of high signal intensity on T1- and T2-weighted images was present inside the mass. Its significance was unknown, perhaps representing neighbouring tissues trapped when the tumour was growing. After intravenous contrast injection, the mass was greatly and heterogeneously enhanced, exhibiting a layered pattern (fig. 2). The tumour's DCE analysis showed a progressive curve without plateau or washout. Regions of interest located in the layers demonstrated the same profile curves of progressive enhancement with variable intensity.

DW-MRI sequences (fig. 3) showed heterogeneous high signal intensity on b0 and b1000 sequences. Typical architecture was only observed on b0 sequences. Apparent 
Brunelle et al.: Aggressive Angiomyxoma with Diffusion-Weighted Magnetic Resonance Imaging and Dynamic Contrast Enhancement: A Case Report and Review of the Literature

diffusion coefficient (ADC) mapping showed a high value in the tumour, estimated at $2 \times 10^{-3}$ $( \pm 0.15) \mathrm{mm}^{2} / \mathrm{sec}$.

Thus, MRI was highly suggestive of AA. It also showed disease extension, notably through the pelvic diaphragm, which made a combined transperineal and laparoscopic abdominopelvic approach necessary. Before surgery, the examination under general anesthesia showed that the mass was soft, renitent, and seemed to come from the pelvic cavity through the levator ani muscles. Tumour excision was monobloc and macroscopically complete, without any rectal or vaginal resection or tumour capsular rupture. The postoperative course was uneventful.

Macroscopic examination of the surgical specimen showed a firm, partly circumscribed tumour measuring $16 \times 12 \times 6.5 \mathrm{~cm}$. A cut section showed a homogeneous, glistening, and gelatinous appearance without any hemorrhagic or necrotic areas. Microscopic analysis showed a monotonous hypocellular proliferation of small nonatypical stellate-shaped cells arranged in a myxoid stroma, along with numerous, variably sized vessels. No mitosis or necrosis could be seen. In its periphery, the proliferation infiltrated the nontumour adipose tissue, and resection margins were focally involved by the tumour cells. Immunohistochemical analysis showed strong expression of CD34, and estrogen and progesterone receptors by tumour cells, without expression of desmin, CD117, $\beta$-catenine, and MDM2, nor epithelial (pancytokeratin, EMA), smooth muscle ( $\alpha$-smooth actin, H-caldesmon), and Schwann cell (PS100) markers. The final diagnosis was pelvi-perineal AA.

After surgery, no adjuvant treatment was given, and the patient was regularly monitored clinically and radiologically. At her last visit, in October 2012, more than 1 year after the diagnosis, the patient was alive with an excellent performance status and without any recurrence of the disease on pelvic DW-MRI.

\section{Discussion}

$\mathrm{AA}$ is a compliant tumour that grows slowly in the perineal or pelvic region and can cross the pelvic diaphragm [5]. Classically, the tumour shows finger-like projections at the periphery and into the surrounding tissue without infiltrating or invading adjacent organs. In general, the tumour is well limited and large (often more than $10 \mathrm{~cm}$ in diameter) at the time of diagnosis, and its consistency is soft or renitent $[1,6]$. Histologically, AA is a benign hypocellular tumour, composed of scattered spindle or stellar cells, embedded in a loose myxoid matrix with a few collagen fibers and abundant vessels of varying sizes. Mitotic figures and necrosis are absent. Infiltration into fat, muscle, and nerves is seen. Frequently, tumour cells show positive immunohistochemical staining for desmin, smooth muscle actin, muscle-specific actin, and hormone receptors. S100 staining is always negative. The pathogenesis of AA is unclear. The true origin is likely mesenchymal. Rare and recent molecular analyses have identified translocation in the 12q13-15 region involving the HMGA2 gene.

Aggressiveness is related to the high rate of local recurrence after surgery because of frequent incomplete excision. This can be due to the absence of a preoperative diagnosis and estimation of the actual extent of the tumour, or to a particular strategic location of the mass near the urethra, vagina, anal sphincter and rectum, with frequent extension through the pelvic diaphragm, making complete resection difficult. Relapses generally occur within 3 years after surgery, but may be more delayed [1]. The formerly high rate of relapse has decreased from $\sim 75$ to $10-30 \%$ during the last 20 years thanks to improved imaging techniques, which allow a more complete surgical resection [6]. AA usually does not 
Brunelle et al.: Aggressive Angiomyxoma with Diffusion-Weighted Magnetic Resonance Imaging and Dynamic Contrast Enhancement: A Case Report and Review of the Literature

metastasize. Only 2 metastatic cases have been reported, including 1 death [3]. AA in men is unusual and affects the genitourinary tract - especially the scrotum and inguinal region [1, 4].

The clinical symptoms are various and nonspecific. They appear slowly due to the growth pattern of the tumour. Most patients present with a slow-growing palpable or visible mass in the pelvi-perineal region. Other signs may include discomfort or pain due to the mass, or signs related to pressure effects on adjacent organs such as dyspareunia and dysuria. There are many differential diagnoses. Clinical examination can rule out infectious or inflammatory disease such as vulvar abscesses. Morphological imaging like computed tomography (CT) checks for the absence of pelvic hernia, gynaecological prolapse or myoma. Non-Hodgkin's lymphoma usually presents with enlarged lymph nodes in other locations. Vaginal cysts, Gartner cysts and Bartholin cysts are frequent and easy differential diagnoses that do not show significant enhancement except if infected. In that case, MRI will usually show peripheral enhancement of the wall of the cyst with variable thickness, which is totally different from the AA pattern of enhancement. The lack of macroscopic fat on CT and MRI sequences with and without fat suppression can rule out infiltrating angiolipoma and lipoma or liposarcoma with or without myxoid component [7, 8]. Endometriomas and melanomas show typical high signal intensity on T1-weighted images without signal intensity dropout on fat-suppressed images. Myxoma is mainly an intramuscular tumour that occurs in older patients, and whose enhancement does not show the same features as AA [8]. Vulval angiomyofibroblastoma is a difficult differential diagnosis because of its similar location and histology. MRI is also a helpful diagnostic tool with in-phase and opposed-phase sequences showing microscopic fat inside the angiomyofibroblastoma. Other diagnoses such as myxoid neurofibroma, myxoid-type malignant fibrous histiocytoma, myxofibrosarcoma, vaginal botryoid pseudosarcoma, and rhabdomyosarcoma are made by histological analysis of the tumour.

In general, MRI will help with the preoperative diagnosis by showing typical images of $\mathrm{AA}$, as in our case. This is all the more important because, if the final diagnosis is obviously based on histology and immunohistochemistry, a preoperative diagnosis may be difficult to obtain from a percutaneous biopsy.

Thus, imaging and particularly MRI are fundamental for the clinical management of AA [2]. Imaging may be highly suggestive of diagnosis if the tumour is large enough. Typical images show the lesion as a well-limited mass, displacing rather than infiltrating adjacent organs and muscles, with content rich in water, loose myxoid matrix, and hypervascularity. Finger-like projections into neighbouring adipose tissue can be present and are relatively characteristic of AA [9]. Classically, US without contrast injection shows a homogeneous and hypoechoic mass, sometimes cystic, that may be misleading [10]. CT scan shows an enhanced mass, which is iso- or hypoattenuating relative to muscle [7, 11]. MRI shows very characteristic aspects: an isointense tumour (more rarely hypointense) relative to muscle on T1-weighted images, and hyperintense on T2-weighted images (high water content and loose myxoid matrix) [2, 5, 7]. The tumour enhances avidly and heterogeneously after intravenous gadolinium contrast administration, reflecting its inherent vasculature $[2,5,7$, 8]. A typical MRI feature of AA is the presence of an internal layered pattern and/or swirled architecture, which is described in $83 \%$ of cases on T2-weighted MRI or on T1-weighted MRI after intravenous contrast injection [2, 5-9]. These aspects reflect the fibrovascular stroma architecture and its enhancement. Our case perfectly illustrated these MRI findings, allowing us to suspect the AA diagnosis before surgery.

Preoperative MRI is also crucial to determine the true extent of the disease and plan the most optimal surgical approach [9]. The importance of accurate preoperative diagnosis and 
extent screening is clearly reported. In our case, an abdominoperineal approach was chosen as the tumour extended above the pelvic diaphragm on MRI, allowing complete resection. During treatment, MRI allows monitoring of the response to eventual preoperative hormone therapy. Finally, MRI is very helpful in order to detect local relapses during follow-up. Recurrent lesions display the same MRI aspect as the primary tumours [2].

Recently, DW imaging (DWI) has become a useful adjunct in oncology. Because of its high sensitivity in detecting tumour presence, its noninvasive nature, and its ability to provide valuable qualitative and quantitative information, DWI has become indispensable for characterizing lesions, and for monitoring the response to nonsurgical therapy and the patient's follow-up after treatment [12]. This is already well documented for the detection of prostate or rectal cancer relapse [12]. To our knowledge, DWI-MRI has not been reported thus far for AA. We report here the first description. The typical architecture of the tumour was seen on T2-weighted images and b0 sequences; however, it disappeared both as the $\mathrm{b}$ value increased and on ADC mapping imaging even in a mass more than $10 \mathrm{~cm}$ in diameter. DWI also showed a high value of tumour ADC measurements in the region of $2 \times 10^{-3}$ $\mathrm{mm}^{2} / \mathrm{sec}$, comparable with ADC calculations in myxoid tumours. The persistence of high tumour signal intensity on DWI with a high $b$ value suggests the possibility of easy and earlier relapse diagnosis using these types of sequences. Furthermore, we can hypothesize that normal MRI using DW sequences during patients' follow-up is probably an effective way to confirm the absence of recurrence. However, AA can theoretically be polymorphous, and more patients in larger case series are needed to confirm these first observations.

Due to the rarity of this disease, treatment recommendations are based on case reports and small series. Wide surgical resection is the treatment of choice, sometimes after preoperative angiographic embolization to decrease the size of the tumour [11]. But, because of its frequently large size and location in close proximity to vital organs (urogenital tract and rectum), wide excision is not always possible and/or may cause significant morbidity in young patients. Furthermore, the prognostic value of extensive surgical resection to obtain clear margins is questioned. A review of over 100 cases published in 2000 by Chan et al. [1] showed that patients with clear resection margins have similar chances of remaining disease free (50\% at 10 years) compared with those having tumourinvolved resection margins (40\%). Later publications confirmed these findings [6,11]. These observations explain that incomplete resection is acceptable nowadays if followed by longterm follow-up and careful monitoring for timely identification and prompt resection of relapse. Treatment involving chemotherapy and radiotherapy has been disappointing, probably due to the low mitotic activity of the tumours $[1,11]$. AA is likely hormone dependent as suggested by the reproductive age of the patients, the few cases reported during pregnancy, and the frequent expression of hormone receptors [6]. Thus, gonadotropin-releasing hormone agonists have been administered to AA patients in the postoperative as well as in the preoperative setting with dramatic responses, facilitating surgery or avoiding mutilating surgery $[10,13,14]$. One case report of AA treated exclusively with gonadotropin-releasing hormone agonists was published in 2003 [13]. Two cases of successful control of recurrent AA with relatively high doses of external radiotherapy have been reported [15], but a literature analysis shows a definite shift towards less morbid treatments.

After treatment, long-term follow-up is required since late recurrences have been described up to 14 years following treatment [1]. Recurrence rates are variable in the literature and probably depend on when patients were treated (related to surgical and MRI quality). Reviewing 106 cases, Chan et al. [1] found a recurrence rate in the region of 50\% between 1984 and 1998. Nowadays, the rate of recurrence is undoubtedly lower. Otherwise, 
Brunelle et al.: Aggressive Angiomyxoma with Diffusion-Weighted Magnetic Resonance Imaging and Dynamic Contrast Enhancement: A Case Report and Review of the Literature

$71 \%$ of recurrences occurred within the first 3 years, regardless of the initial tumour size and patient's age [1]. Periodic clinical examination may be insufficient and imaging studies, such as MRI, are fundamental. They may detect recurrence early, when the tumour is smaller in size, making surgery easier to perform or allowing the use of less morbid percutaneous ablative therapeutic strategies such as radiofrequency ablation or cryotherapy.

\section{Conclusion}

MRI is crucial in the management of AA patients for its diagnostic contribution and preoperative assessment of the actual extent of the tumour. During eventual neoadjuvant treatment, it allows monitoring of the tumour response and, after treatment, it is very useful for detecting recurrences.

Here, we presented the use of MRI in a typical case of AA. However, this case report is also the first description in the literature of AA with complete DCE and DWI analysis. We have updated the current knowledge on AA and, furthermore, we recommend the use of diffusion sequences in the follow-up of young patients treated for AA, regardless of what type of treatment they have previously had.

\section{References}

$\checkmark 1$ Chan YM, Hon E, Ngai SW, Ng TY, Wong LC: Aggressive angiomyxoma in females: is radical resection the only option? Acta Obstet Gynecol Scand 2000;79:216-220.

-2 Jeyadevan NN, Sohaib SA, Thomas JM, Jeyarajah A, Shepherd JH, Fisher C: Imaging features of aggressive angiomyxoma. Clin Radiol 2003;58:157-162.

-3 Blandamura S, Cruz J, Faure VL, Machado PI, Ninfo V: Aggressive angiomyxoma: a second case of metastasis with patient's death. Hum Pathol 2003;34:1072-1074.

4 Haldar K, Martinek IE, Kehoe S: Aggressive angiomyxoma: a case series and literature review. Eur J Surg Oncol 2010;36:335-339.

-5 Outwater EK, Marchetto BE, Wagner BJ, Siegelman ES: Aggressive angiomyxoma: findings on CT and MR imaging. AJR Am J Roentgenol 1999;172:435-438.

6 Fetsch JF, Laskin WB, Lefkowitz M, Kindblom LG, Meis-Kindblom JM: Aggressive angiomyxoma: a clinicopathologic study of 29 female patients. Cancer 1996;78:79-90.

7 Sinha R, Verma R: Case 106: aggressive angiomyxoma. Radiology 2007;242:625-627.

8 Stewart ST, McCarthy SM: Case 77: aggressive angiomyxoma. Radiology 2004;233:697-700.

-9 Wiser A, Korach J, Gotlieb WH, Fridman E, Apter S, Ben-Baruch G: Importance of accurate preoperative diagnosis in the management of aggressive angiomyxoma: report of three cases and review of the literature. Abdom Imaging 2006;31:383-386.

$\rightarrow 10$ Giles DL, Liu PT, Lidner TK, Magtibay PM: Treatment of aggressive angiomyxoma with aromatase inhibitor prior to surgical resection. Int J Gynecol Cancer 2008;18:375-379.

11 Han-Geurts IJ, van Geel AN, van DL, Bakker Md, Eggermont AM, Verhoef C: Aggressive angiomyxoma: multimodality treatments can avoid mutilating surgery. Eur J Surg Oncol 2006;32:1217-1221.

12 Malayeri AA, El Khouli RH, Zaheer A, Jacobs MA, Corona-Villalobos CP, Kamel IR, Macura KJ: Principles and applications of diffusion-weighted imaging in cancer detection, staging, and treatment follow-up. Radiographics 2011;31:1773-1791.

13 Poirier M, Fraser R, Meterissian S: Case 1. Aggressive angiomyxoma of the pelvis: response to luteinizing hormone-releasing hormone agonist. J Clin Oncol 2003;21:3535-3536.

14 Shinohara N, Nonomura K, Ishikawa S, Seki H, Koyanagi T: Medical management of recurrent aggressive angiomyxoma with gonadotropin-releasing hormone agonist. Int J Urol 2004;11:432-435.

15 Suleiman M, Duc C, Ritz S, Bieri S: Pelvic excision of large aggressive angiomyxoma in a woman: irradiation for recurrent disease. Int J Gynecol Cancer 2006;16(suppl 1):356-360. 


\section{Case Reports in Oncology}
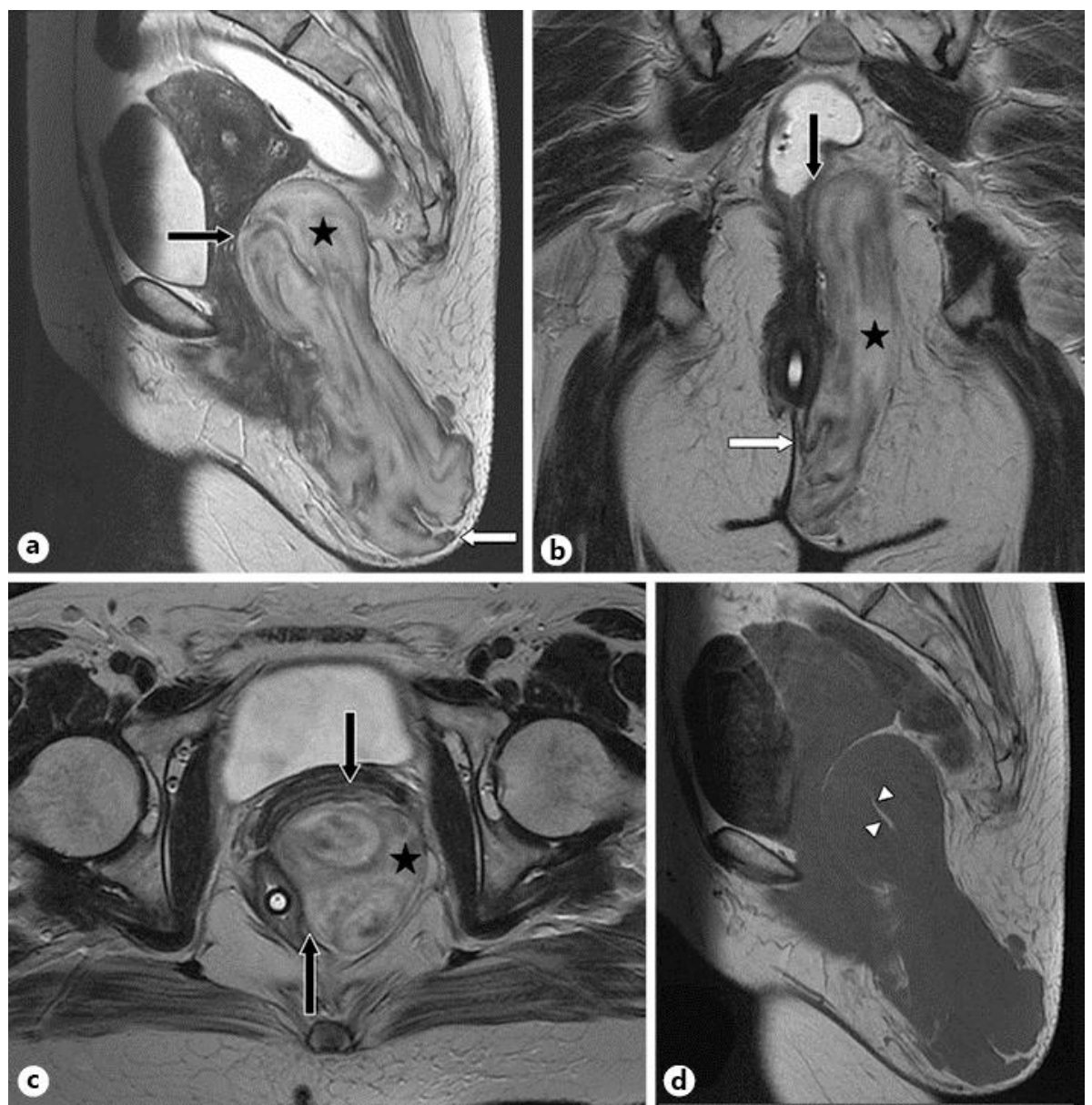

Fig. 1. Sagittal (a), coronal (b), and axial (c) T2-weighted MR images in a 51-year-old woman with AA show a bulky perineal and intrapelvic tumour displacing rather than infiltrating the vagina, anal canal, and rectal structures (black arrows). The mass presents typical high signal intensity with layered strands of lower signal intensity and swirled architecture (stars). Finger-like projections into the underlying adipose tissue can be seen (white arrows). d Sagittal T1-weighted MR image shows a homogeneous tumour, isointense relative to muscle, with a fat linear structure of high signal intensity inside (arrowheads). 


\section{Case Reports in Oncology}

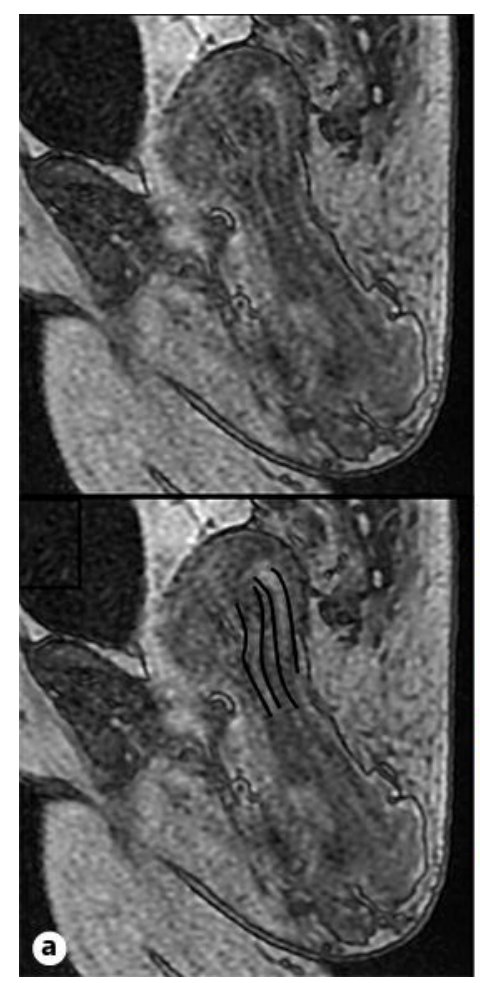

\begin{tabular}{l|l}
\hline Case Rep Oncol 2013;6:373-381 \\
\hline DOI: $10.1159 / 000353487$ & $\begin{array}{l}\text { C 2013 S. Karger AG, Basel } \\
\text { www.karger.com/cro }\end{array}$ \\
\hline
\end{tabular}

Brunelle et al.: Aggressive Angiomyxoma with Diffusion-Weighted Magnetic Resonance Imaging and Dynamic Contrast Enhancement: A Case Report and Review of the Literature
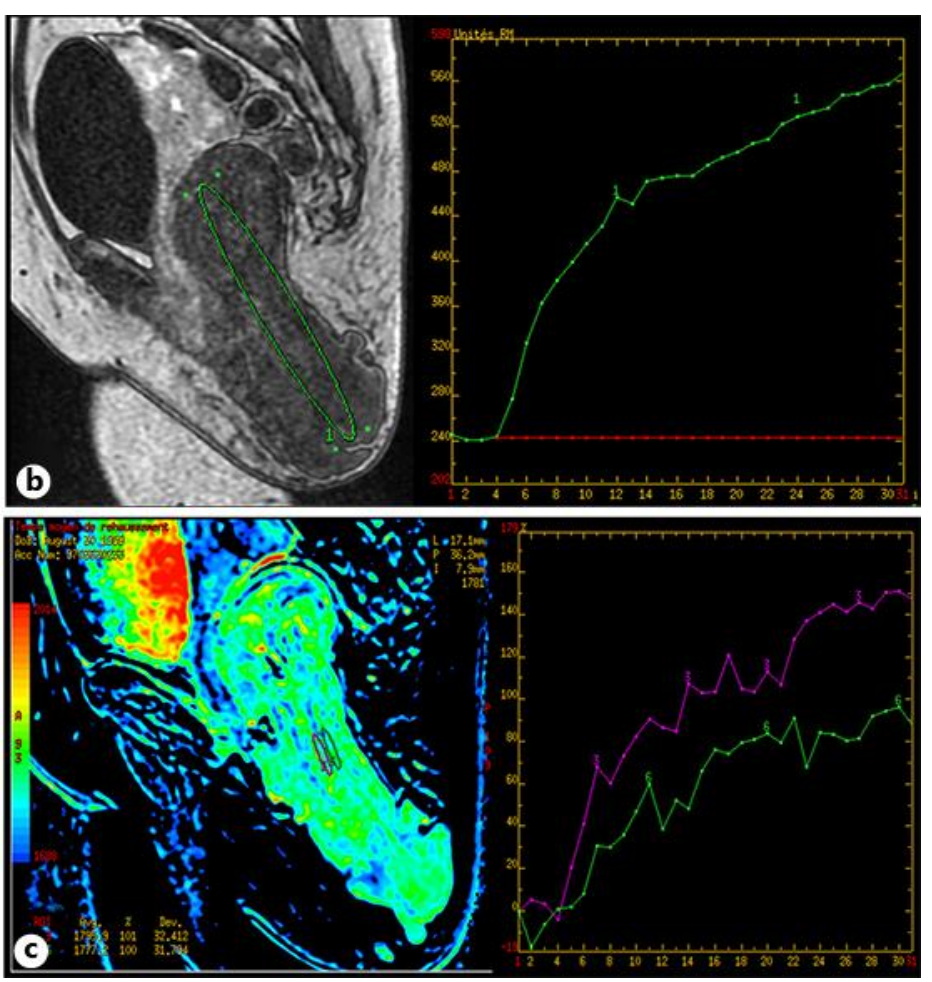

Fig. 2. Sagittal T1-weighted MR imaging after intravenous contrast injection with $0.1 \mathrm{mmol} / \mathrm{kg}$ gadoteric acid (Gd-DOTA, Dotarem, Guerbet, Roissy, France) at $2.5 \mathrm{ml} / \mathrm{s}$ in a 51-year-old woman with AA. a The mass presents a heterogeneous enhancement with layered architecture (black lines). b, c The tumour's DCE analysis shows a progressive curve without plateau or washout. DCE mapping shows the same layered pattern. Regions of interest located in the layers demonstrate the same profile curves of progressive enhancement with variable intensity. 


\section{Case Reports in Oncology}

\begin{tabular}{l|l}
\hline \multicolumn{2}{l}{ Case Rep Oncol 2013;6:373-381 } \\
\hline DOI: $10.1159 / 000353487$ & $\begin{array}{l}\text { C 2013 S. Karger AG, Basel } \\
\text { www.karger.com/cro }\end{array}$ \\
\hline
\end{tabular}

Brunelle et al.: Aggressive Angiomyxoma with Diffusion-Weighted Magnetic Resonance Imaging and Dynamic Contrast Enhancement: A Case Report and Review of the Literature
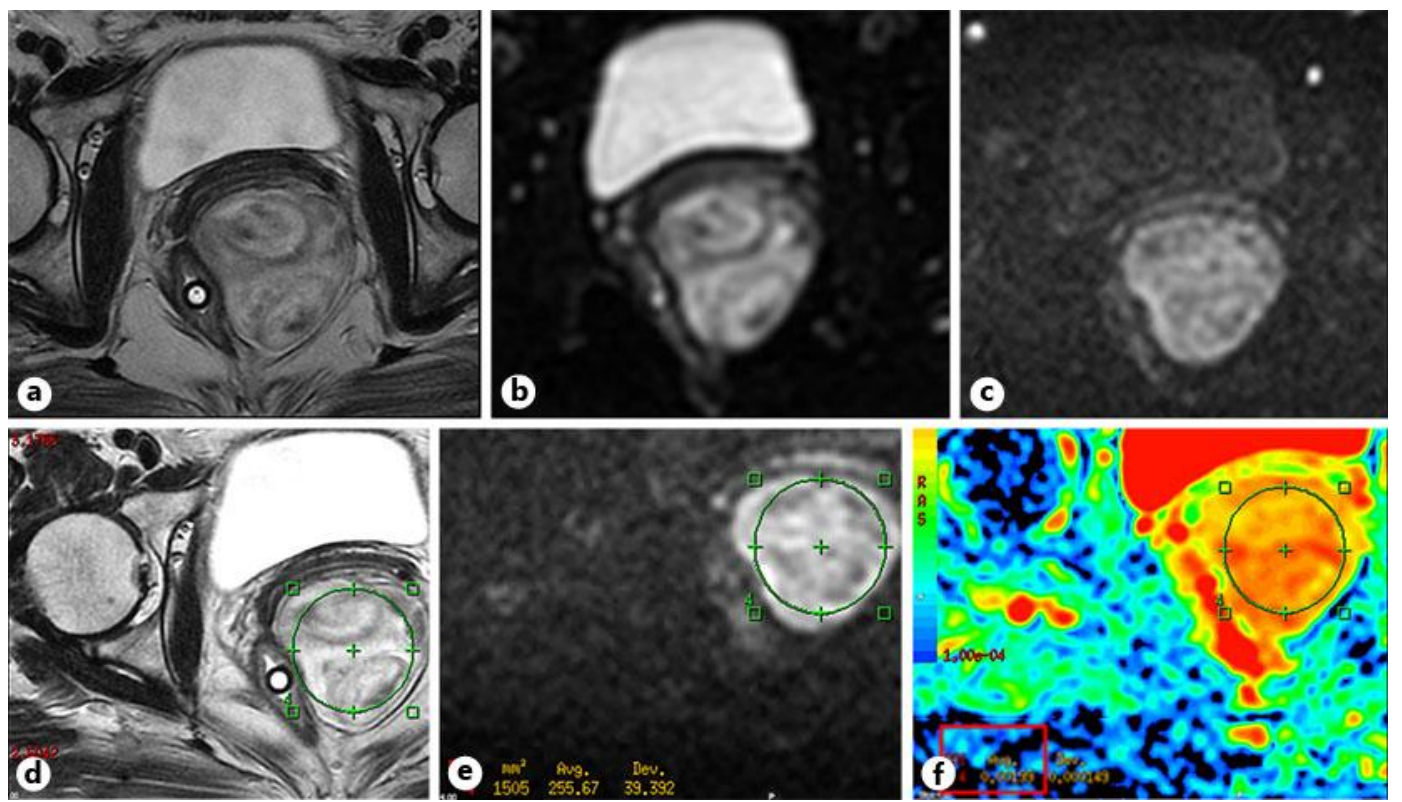

Fig. 3. Axial T2-weighted MR image (a, d) and axial DWI (b, c, e) in a 51-year-old woman with AA. DWI sequences show heterogeneous high signal intensity on b0 (b) and b1000 (c, e) sequences. Typical architecture is only observed on b0 sequences. $\mathbf{f}$ ADC mapping shows a high value in the tumour, and ADC calculations are estimated at $2 \times 10^{-3}( \pm 0.15) \mathrm{mm}^{2} / \mathrm{sec}$. 